\title{
The effects of verbal feedback of elicited heart rate changes on subsequent voluntary control of heart rate
}

\author{
ALAN WRIGHT, DOUGLAS CARROLL, and COLIN V. NEWMAN \\ University of Birmingham, P. O. Box 363, Birmingham B15 2TT, England
}

Three groups of 10 subjects were employed to determine the effect of verbally labeling elicited heart rate (HR) changes upon subsequent voluntary HR control. While one group (VF group) received veridical feedback when criterion HR increases were elicited by loud tones, a second, reverse feedback (RF) group were falsely informed that their HR had decreased in such circumstances. A control (C) group received no feedback regarding the nature of elicited responses. Groups VF and C were subsequently required to increase HR, while Group RF was instructed to decrease HR. The VF group displayed a tendency toward greater accelerative changes than either of the other groups. Only in the VF group was HR increase a consistent outcome of instructions.

Although voluntary acceleration and deceleration of heart rate (HR) has now been amply demonstrated in human subjects, speculation as to the processes underlying such cardiac adjustments has been rather a minority concern. Recently, however, Brener (1974a, 1974b) proposed that James' (1890) ideo-motor theory might usefully be elaborated to account for voluntary cardiovascular control. Brener postulated that, for example, an instruction to raise HR activates a neural response image embodying a representation of the interoceptive associates of the increased HR. Such an instruction acquires this activating facility through the prior association of its descriptive terms with the interoceptive events contemporary with HR increase. This association of an exteroceptive description with a particular pattern of interoceptive stimulation Brener terms "calibration." Finally, according to Brener's proposal, prior to the establishment of voluntary control over a response such as HR increase, that response must be elicited in an involuntary, reflexive fashion. In the usual scheme of events, presumably, the calibration of exteroceptive and interoceptive stimuli occurs during such reflexively elicited activity.

Thus it was hypothesized, on the basis of Brener's (1974a, 1974b) speculation, that verbally informing subjects of elicited HR changes as they occurred should constitute the necessary condition for the operation of the calibration procedure. Calibration, in turn, should allow subjects to emit these HR changes upon the receipt of instructions which included the semantic elements of the previous verbal information. Thus, subjects having elicited HR changes verbally indexed in this way should demonstrate superior HR control to subjects not informed about the nature of their HR reactions. Further, it might be argued from Brener's model that only the consistency, and not the veracity, of the feedback should be of real consequence: for example, naive subjects could be informed that their HRs were decelerating, when in fact HR had increased. When asked subsequently to lower HR, such subjects should demonstrate an increase, that is, instructions to decrease HR should activate the response image associated with the exteroceptive descriptor "HR down." However, the descriptor in this instance would have been systematically paired with the interoceptive associates of HR increase.

\section{METHOD}

There were 30 subjects ( 20 male, 10 female) of various occupations and ranging in age from 17 to 32 years (median age $=$ 22 years). None of the subjects had any previous experience of HR control studies, meditation, or yoga, nor suffered from any cardiac disorders. Each subject was paid $\$ 1.00$ for participation in the experiment, which lasted approximately $90 \mathrm{~min}$. The subjects were randomly allocated to one of three groups with the provision of approximately equal sex representation. The groups were designated veridical feedback (VF), reverse feedback (RF), and control (C).

HR and respiration rate (RR) were recorded on a Beckman Type $\mathrm{R}$ dynograph. HR was measured using Beckman $\mathrm{AG} / \mathrm{AgCl}$ skin electrodes, attached to the region just above the left ankle bone and the right wrist using Beckman electrolyte paste, and recorded using a Beckman 9857 cardiotachometer coupler. The pulse output from the cardiotachometer was relayed to a PDP-11 computer, via an A-D converter, permitting the accurate measurement of each interbeat interval. RR was monitored using a thermistor secured beneath the subject's left nostril. Two further "dummy" electrodes were attached to the right forearm.

The length and interval between a series of tones used to elicit HR changes was controlled by a Tandberg 2341 tape recorder and a multi-X student box. Tone production was by a Farnell oscillator, which fed into headphones via a noise-free switch, and an amplifier producing a $1-\sec 1,000-\mathrm{Hz}$ tone of $100 \mathrm{~dB}$.

Subjects semi-reclined on an adjustable divan in a soundattenuated temperature-controlled room. Subjects were told that the experiment was concerned initially with individual differences in HR response to a variety of stimuli. 
During electrode attachment subjects in Groups VF and RF were informed that verbal feedback, via an intercom, would be given regarding the direction of their HR responses. Subjects in Group C received no such instructions. The "dummy" forearm electrodes were represented as a vehicle for the delivery of moderately painful, but harmless, electric shocks.

Following 10 min relaxation, all subjects were presented with a series of 100-dB tones. Raskin, Kotses, and Bever (1969) indicated that auditory stimuli should, initially at least, elicit HR acceleration. In all, 40 such tones were presented at random intervals ranging from 15 to $25 \mathrm{sec}$ (mean interval $=20 \mathrm{sec}$ ). Subjects had been informed that immediately after any of the first 10 tones they might receive an electric shock. However, no shocks were delivered. The threat of shock was employed to retard the habituation of the initial $H R$ acceleration response. Carroll and Pokora (1976) have reported that threat of impending shock serves to retard the rate of skin conductance response habituation. For the final 30 tones the threat of shock was lifted, and subjects were required to press a reaction time button following each tone. It was assumed that this further conferment of signal significance on the tones would optimize the appearance of HR acceleration to the tones.

During tone presentation, subjects in the VF and RF groups were given feedback of HR accelerations of 10 or more beats/min, persisting for at least 3 beats, as indicated by the cardiotachometer record. For Group VF, any such tone-elicited HR accelerations, or occasional spontaneous accelerations not adjudged to reflect gross movements or respiratory cycling effects and which met the criteria, were immediately labeled "HR up." RF subjects, on the other hand, had such changes falsely labeled as "HR down." Group C received neither veridical nor reverse feedback.

Following tone presentations, subjects were asked to attempt to change their HR. Subjects in the VF and C groups were asked to raise their HRs, while subjects in Group RF were instructed to decelerate HR. Subjects were instructed not to alter their breathing and to refrain from moving or tensing their muscles during attempted HR control. Subjects were given $20 \mathrm{HR}$ control trials of 1-min duration, each separated by a rest period of $1 \mathrm{~min}$. Timing and signaling of trials was effected by the PDP-11 computer. No feedback of any description was given during this phase of the experiment.

Reduction of the initial HR data was performed by the PDP-11, which for each trial period printed out the mean HR in beats per minute (bpm) during the trial and the mean HR for the 20 -sec portion of the intertrial interval immediately preceding trial onset. Pretrial and trial HR change scores were then computed and the overall mean HR change score calculated for each subject. $R R$ data in cycles per minute (cpm) were similarly scored and reduced.

\section{RESULTS}

The average HR change scores in bpm for the three groups were: $\mathrm{VF},+1.5(\mathrm{SD}=1.39)$; $\mathrm{RF},-.72(\mathrm{SD}=$ $1.61)$; and $\mathrm{C},+.16(\mathrm{SD}=1.59)$. Analysis of variance showed a significant difference between the groups in their HR change scores $[F(2,27)=4.82, p<.05]$. Further exploration of group differences using a Duncan's multiple range test indicated that Groups $C$ and $R F$ were not significantly different from each other $(Q=1.72)$ but that the HR change scores of Groups VF and RF differed $(Q=4.36, p<.01]$. Further, there was a marked tendency for Group VF to demonstrate a HR acceleration performance superior to that of Group $\mathrm{C}$ $(\mathrm{Q}=2.64, \mathrm{p}<.10)$.

While the binomial test indicated that HR acceleration was a fairly consistent result of instructions to increase HR $(x=2, p=.055)$ for the VF group, no consistent directionality of HR change occurred for the C group. For the RF group 7 of the 10 subjects demonstrated HR decrease. The probability associated with this pattern was fairly large $(x=3, p=.172)$.

The average RR change scores in cpm for each of the three groups were: $\mathrm{VF},+.91(\mathrm{SD}=1.22) ; \mathrm{RF},+.18$ $(\mathrm{SD}=1.30) ; \mathrm{C},+1.39(\mathrm{SD}=1.42)$. Analysis of variance computed on these data indicated that no significant group differences in $R R$ change scores existed $[\mathrm{F}(2,27)=1.91]$.

Finally, the VF group received, on average, 22.8 $(\mathrm{SD}=12.9)$ "HR up" labels and the RF group received $20.0(\mathrm{SD}=10.4)$ "HR down" labels.

\section{DISCUSSION}

The present results indicate that the VF group tended to display greater HR accelerations than both the RF and $C$ groups. Further, only in the VF condition was HR increase a consistent outcome of instructions to increase HR. While Group C exhibited no consistency with regard to the direction of exhibited HR changes, Group RF, if anything, showed a marginal tendency to comply with the instructions to decrease HR. No significant group differences in RR were detected; all groups showed a trend toward increased RR during the HR control trials. This result appears to indicate that such $H R$ changes as occurred did so relatively independently of RR changes.

The observation that subjects given constant and veridical information of elicited HR increases subseqently tend to achieve superior voluntary increases than do subjects not so provided is in keeping with Brener's (1974a, 1974b) account of voluntary cardiovascular control. Calibration of the interoceptive associates of elicited HR increases in terms of verbal labels permitted the later instructions, semantically consistent with these labels, to activate the central response image representation of such interoceptive stimuli. However, the behavior of the RF group is difficult to reconcile with Brener's model, which would argue that the veracity of verbal feedback should be of no real consequence and that subjects informed that elicited HR increases were in fact HR decreases should, when subsequently requested to decrease HR, comply by effecting an increase. This clearly did not occur. It might be argued that subjects in Group RF already possessed response images of cardiac variations calibrated in terms of more usual vocabulary, and that the present relatively modest procedures aimed at promoting a recalibration were simply insufficient for this purpose. Clearly, the impact of a more protracted labeling enterprise would be instructive.

\section{REFERENCES}

Brener, J. A general model of voluntary control applied to the phenomena of learned cardiovascular change. In P. A. Obrist, A. H. Black, J. Brener, \& L. V. DiCara (Eds.), Cardiovascular psychophysiology. Chicago: Aldine-Atherton, 1974. (a)

BRENER, J. Factors influencing the specificity of voluntary cardiovascular change. In L. V. DiCara (Ed.), Limbic and autonomic nervous systems research. New York: Plenum, 1974. (b)

Carroll, D., \& Pokora, J. The effects of threat of shock on SCR habituation to simple auditory stimuli. Physiological Psychology, 1976, 4, 94-96.

James, W. Principles of psychology. New York: Holt, 1890.

RAskin, D. C., Kotses, H., \& BeVER, J. Autonomic indicators of orienting and defensive reflexes. Journal of Experimental Psychology, 1969, 80, 423-433.

(Received for publication May 16, 1977.) 